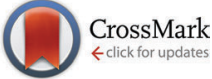

Cite this: Phys. Chem. Chem. Phys. 2016, 18, 18880

Received 1st June 2016, Accepted 20th June 2016

DOI: $10.1039 / \mathrm{c} 6 \mathrm{cp} 03808 \mathrm{e}$

www.rsc.org/pccp

\section{Magnetic response properties of gaudiene - a cavernous and aromatic carbocage + t}

\begin{abstract}
M. Rauhalahti, ${ }^{a}$ A. Muñoz-Castro* ${ }^{b}$ and D. Sundholm*a
A spherical and cavernous carbocage molecule exhibiting faces with larger ring sizes than regular fullerenes is a suitable species for investigating how molecular magnetic properties depend on the structure of the molecular framework. The studied all-carbon gaudiene $\left(\mathrm{C}_{72}\right)$ is a highly symmetrical molecule with three- and four-fold faces formed by twelve membered rings. Here, we attempt to unravel the magnetic response properties of $\mathrm{C}_{72}$ by performing magnetic shielding and current density calculations with the external magnetic field applied in different directions. The obtained results indicate that the induced current density flows mainly along the chemical bonds that are largely perpendicular to the magnetic field direction. However, the overall current strength for different directions of the magnetic field is nearly isotropic differing by only $10 \%$ indicating that $C_{72}$ can to some extent be considered to be a spherical aromatic molecule, whose current density and magnetic shielding are ideally completely isotropic. The induced magnetic field is found to exhibit long-range shielding cones in the field direction with a small deshielding region located perpendicularly to the field outside the molecule. The magnetic shielding is isotropic inside the molecular framework of $C_{72}$, whereas an orientation-dependent magnetic response appears mainly at the exterior of the molecular cage.
\end{abstract}

\section{Introduction}

The since the seminal discovery of buckminsterfullerene $\left(\mathrm{C}_{60}\right),{ }^{1-3}$ rapid and extensive development has happened in the field of carbon nanostructures, leading to a variety of interdisciplinary applications of technological interest. ${ }^{4-7}$ The almost spherical $\mathrm{C}_{60}$ belongs to the icosahedral $\left(I_{\mathrm{h}}\right)$ point group. It has sixty chemically equivalent $\mathrm{sp}^{2}$ carbon atoms representing the basic motif for hollow all-carbon materials that have technologically relevant properties such as high specific surface area, efficient electron acceptor properties, good chemical stability, and an available hollow interior. ${ }^{4-6,8}$ The hollow structure makes fullerenes of interest for a wide range of applications such as in lithium-ion batteries, ${ }^{9-11}$ catalyst supports, ${ }^{12,13}$ hydrogen storage, ${ }^{14}$ as electron acceptors in solar cells, ${ }^{15}$ and as carrier molecules for drug and gene delivery, ${ }^{16}$ among others.

Extensive research efforts, whose aim is to develop, functionalize and characterize novel carbon structures, have been motivated by the wish to design molecules and materials with

\footnotetext{
${ }^{a}$ Department of Chemistry, University of Helsinki, P.O. Box 55 (A. I. Virtanens Plats 1), FIN-00014, Finland. E-mail: sundholm@chem.helsinki.fi

${ }^{b}$ Laboratorio de Química Inorgánica y Materiales Moleculares, Universidad Autonoma de Chile, El Llano Subercaseaux 2801, Santiago, Chile.

E-mail: alvaro.munoz@uautonoma.cl

$\dagger$ In memory of Sir Prof. Harold W. Kroto, for his insightful discoveries.

\$ Electronic supplementary information (ESI) available: $\pi$-Electron levels and shielding cone representation for 2 and 3. See DOI: 10.1039/c6cp03808e
}

important technological properties. Recently, the seminal construction of a hollow all-carbon molecule $\left(\mathrm{C}_{72}\right)$ was proposed on the basis of one of the architect Gaudís artifacts. ${ }^{17}$ The molecule is therefore called gaudiene (1). This carbon structure belonging to the octahedral $\left(O_{\mathrm{h}}\right)$ point group has twelvemembered rings with radii of $2.1 \AA$ and $2.4 \AA$ around the threeand four-fold symmetry axes of the $O_{\mathrm{h}}$ point group, respectively. Larger gaudiene structures can formally be obtained by wrapping graphyne sheets onto a sphere leading to hollow all-carbon structures with alternating $\mathrm{sp}^{2}$ and $\mathrm{sp}$ hybridized carbons atoms as compared to the $\mathrm{sp}^{2}$ hybridization of the carbons in fullerenes. ${ }^{18}$

Gaudiene $\left(\mathrm{C}_{72}\right)$ exhibits 72 vertices leading to the same number of $\pi$ electrons on the surface of the highly symmetrical cage $\left(O_{\mathrm{h}}\right)$. The $72 \pi$ electrons fulfill Hirsch's $2(n+1)^{2}$ rule for spherical aromatic species, with $n=5 .^{19-22}$ The aromatic properties of $\mathrm{C}_{72}$ were confirmed by calculating the strength of the magnetically induced current density susceptibility around the molecule using the gauge-including magnetically induced current (GIMIC) method. ${ }^{17,23-25}$ The magnetically induced current susceptibility is here analyzed in order to extend our understanding of the orientation-dependent character ${ }^{26}$ of the magnetic response of the cavernous and almost spherical carbon structure. We investigate the magnetic shielding functions that yield the magnetic response in the vicinity of the molecule, as given by induced magnetic field $\left(B^{\text {ind }}\right) \cdot{ }^{27-29}$ The visualization of $B^{\text {ind }}$ offers an overall picture of the magnetic shielding and deshielding regions around the molecule, and has also been widely used for 
demonstrating ring current effects in aromatic molecules and to graphically show the anisotropic effects that originate from molecular substituents. ${ }^{30-35}$

The magnetic response of the spherical aromatic counterpart of $\mathrm{C}_{60}$, namely $\mathrm{C}_{60}{ }^{10+}$, for a given orientation of the applied field revealed that the current density susceptibility of spherical aromatic molecules is almost isotropic around the molecular cage. $^{26,31}$ The uniform shell of diatropic current density susceptibility leads to a strongly shielded region inside the molecular cage. Gaudiene may be another class of spherical aromatic molecules, because magnetic fields are also shielded in the interior of the molecule. ${ }^{17} \mathrm{C}_{72}$ has larger molecular rings and a lower symmetry than $\mathrm{C}_{60}$. It has two different symmetry axes, namely through the three- and four-fold molecular rings of the cage faces. Thus, $\mathrm{C}_{72}$ is an interesting molecule for studies of the magnetic response of almost spherical molecular cages, whose structure significantly differs from fullerenes.

Here, we investigate the magnetic properties of gaudiene by exposing the molecule to an external magnetic field in the three Cartesian directions with the aim to obtain a deeper understanding of the magnetic response of cavernous structures. The analysis of the magnetic response is based on calculations for the free-standing molecular rings of the cage structure, namely the hexadehydro[12]annulene (2) and tetradehydro[12]annulene (3) molecules. The magnetic response is also evaluated by calculating the current density susceptibility using the gauge including magnetically induced current (GIMIC) method..$^{23-25}$ The results of the current susceptibility calculations are compared with the induced magnetic field calculated at discrete points around the molecule. ${ }^{29}$ Thus, two complementary approaches have been employed for understanding the magnetic behavior of gaudiene.

\section{Computational details}

The molecular structure optimizations were performed at the density functional theory (DFT) level employing the ADF code. ${ }^{36,37}$ We used the all-electron triple- $\zeta$ Slater basis set augmented with double polarization functions (STO-TZ2P) and the non-local Becke-Perdew (BP86) functional within the generalized gradient approximation (GGA). ${ }^{38-40}$ The nuclear magnetic shielding constants and nucleus-independent shielding tensors were calculated with the NMR module of ADF at the DFT BP86 level using the STO-TZ2P basis set employing gauge-including atomic orbitals (GIAO). ${ }^{29,41-43}$ In the NMR calculations we used a three-dimensional grid of $41 \times 41 \times 41$ with a separation of $0.635 \AA$.

The magnetically induced current density susceptibilities were calculated using the gauge-including magnetically induced current (GIMIC) method..$^{23-25}$ In the GIMIC calculations, the input information comprises basis set data and the unperturbed and first order magnetically perturbed density matrices obtained from the Kohn-Sham and NMR calculations, respectively. The density matrices were obtained at the BP86/def2-TZVP level in combination with the resolution of the identity (RI) approximation using the Turbomole code version 7.0. ${ }^{44-48}$ Current strengths and current pathways were obtained via numerical integration of the current density susceptibility passing chosen planar domains, cutting chemical bonds at the bond center perpendicularly to the bond. For the molecules consisting of a single ring, the rectangular integration grid was extended from the center of the ring to $5 \AA$ above and below the ring plane and 5 Å outwards from the bond. In gaudiene, the integrations were performed using a circular grid around the chemical bond with a radius of $2.3 \AA$ A. For simplicity, we use the term current density instead of current density susceptibility in the discussion of the results.

\section{Results and discussion}

Gaudiene (1) shown in Fig. 1 has a cavernous structure consisting of six four-fold rings with triple bonds in the center of the edges and eight three-fold faces with alternating double, single, and triple bonds. The resulting all-carbon octahedral structure has annelated hexadehydro[12] annulene (2) and tetradehydro[12]annulene (3) rings leading to a hollow structure with $72 \pi$ electrons on the surface. The neutral isolated ring fragments 2 and 3 with $12 \pi$ electrons are expected to be antiaromatic according to Hückel's 4 n electron count rule, which is also confirmed by calculations of the magnetic shieldings and current densities. ${ }^{49,50}$ The schematic representation of the energy levels of the $\pi$ electrons is given in the ESI. $\neq$ The molecular orbital diagram shows that the freestanding molecule corresponding to the four-fold ring 3 has an open-shell character since it has $14 \pi$-electrons and belongs to the $D_{4 \mathrm{~h}}$ point group, where as 2 belongs to the $D_{3 \mathrm{~h}}$ point group and is a closed-shell molecule. Aromatic rings with 10 or $14 \pi$-electrons are obtained when removing two electrons or by adding two electrons. The doubly cationic species of 2 and 3 with $10 \pi$-electrons, i.e., $2^{2+}$ and $3^{2+}$, have bond distances of $1.241 \AA$ and $1.244 \AA$ for the triple bonds $(\mathrm{C} \equiv \mathrm{C})$, respectively. The lengths of the formal single bonds between the sp and $\mathrm{sp}^{2}$ hybridized carbons $(\mathrm{C}-\mathrm{C})$ are $1.374 \AA$ and $1.401 \AA$ for $2^{2+}$ and $3^{2+}$, respectively. The $\mathrm{C}=\mathrm{C}$ double bonds for $2^{2+}$ are $1.413 \AA$ long. For the neutral and antiaromatic 2 , the $\mathrm{C} \equiv \mathrm{C}$ bond lengths are $1.222 \AA$ as compared to the lengths of the $\mathrm{C}-\mathrm{C}$ bonds of $1.415 \AA$. Thus, the neutral and antiaromatic 2 has a somewhat larger bond-length alternation than the doubly charged and aromatic $2^{2+}$. The dianionic form of $3\left(3^{2-}\right)$ is an aromatic ring with $14 \pi$-electrons. The $\mathrm{C} \equiv \mathrm{C}$ and $\mathrm{C}-\mathrm{C}$ distances are 1.253 $\AA$ and $1.401 \AA$, respectively, which are similar to the bond distances of $3^{2+}$.

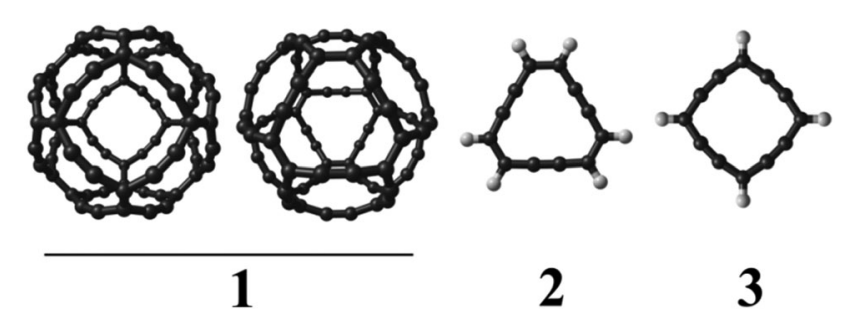

Fig. 1 Schematic representation of the studied compounds. Two different views of gaudiene (1) and the respective molecular versions of its faces (2 and $\mathbf{3}$ ). 
To evaluate the induced magnetic field $\left(B^{\text {ind }}\right)$ in the vicinity of the molecules when exposing them to an external magnetic field $\left(B^{\text {ext }}\right)$, we have calculated the nucleus independent shielding tensor $\left(\sigma_{i j}\right)$ on a grid to obtain a visual representation of $B^{\text {ind }},{ }^{27,28,51}$ which is related to the magnetic shielding tensor through $B_{i}^{\text {ind }}=-\sigma_{i j} B_{j}^{\text {ext }} \cdot{ }^{29}$ Here, indices $i$ and $j$ correspond to the $x$-, $y$ - and $z$-axis of the molecule-fixed Cartesian coordinate system $(i, j=x, y$, and $z)$. The magnetic response property is directly related to the experimentally accessible magnetic chemical shift tensor at the nuclei $\left(\delta_{i j}=-\sigma_{i j}\right)$, which has been widely used for evaluating the aromatic character of molecular rings according to the magnetic criterion. ${ }^{19,22,27,29,52,53}$ The magnetic criterion for aromaticity states that aromatic rings sustain a diatropic ring current when exposed to an external magnetic field. The diatropic ring current leads to a shielding of the magnetic field inside the ring, whereas the exterior parts of the ring $e . g$., the outer hydrogens are deshielded. In contrast, antiaromatic rings sustain a paratropic ring current leading to a strengthening (deshielding) of the magnetic field inside the molecular ring. The isotropic component shown in Fig. 2 corresponds to the averaged magnetic response when exposing the molecule to an external magnetic field $\left(\delta_{\text {iso }}=\left(\delta_{x x}+\delta_{y y}+\delta_{z z}\right) / 3\right)$. The isotropic magnetic shielding or chemical shift accounts for the experimental molecular tumbling. ${ }^{29}$ The calculated $\delta_{\text {iso }}$ for neutral 2 indicates that it is antiaromatic as is also observed experimentally. ${ }^{49,50,54}$ Previous magnetic shielding and current density calculations showed that 2 is antiaromatic. ${ }^{49,50}$ The magnetic shielding function has a deshielding region in the interior of the ring and the experimental ${ }^{1} \mathrm{H}$ NMR chemical shift of 5.55 ppm suggests that the molecular ring sustains a net paratropic ring current. ${ }^{54}$ Calculations of the magnetic shielding function for the doubly charged cation $\left(2^{2+}\right)$ with $10 \pi$ electrons yield a magnetic response that is typical for aromatic rings, which is also in agreement with the expectation from Hückel's $\pi$-electron count rule. The doubly charged tetradehydro[12]annulenes $3^{2+}$ and $3^{2-}$ with 10 and $14 \pi$-electrons, respectively, are also found to be aromatic.

The isosurface representation of $\delta_{\text {iso }}$ at $\pm 5 \mathrm{ppm}$ shown in Fig. 2 reveals that the shielding cones for $2^{2+}$ and $3^{2+}$ are similar to the one obtained for benzene, which is provided as ESI. + The shielding cones are very similar even though $2^{2+}$ and $3^{2+}$ have much larger ring radii of $2.1 \AA$ and $2.4 \AA$, respectively, as

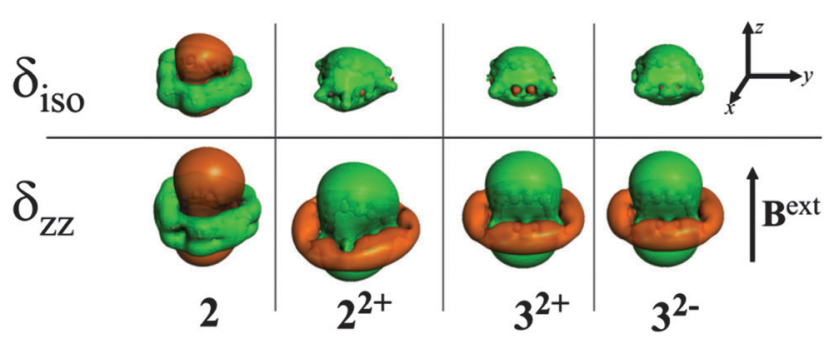

Fig. 2 The magnetic shielding functions for the molecular systems representing the rings in the gaudiene faces. The isosurface value $\pm 5 \mathrm{ppm}$ has been used in the plot. Positive values of the shielding functions are indicated in orange and the negative ones are green. compared to $1.4 \AA$ for the benzene ring. The shielding region for $2^{2+}$ and $3^{2+}$ with a plotting threshold of $\delta=-5$ ppm extends to $2.9 \AA$ above the molecular plane as compared to $2.0 \AA$ for benzene. For $3^{2-}$, which is also aromatic with $14 \pi$ electrons, the isosurface of the magnetic shielding function with a plotting threshold of $\delta=-5$ ppm extends to $3.8 \AA$ above the ring. Thus, for larger molecular rings, the magnetic shielding is more long-range, as one also can expect when applying the classical Biot-Savart law for current loops.

A more thorough understanding of the magnetic response can be obtained by analyzing different components of the NMR chemical shift tensor $\delta_{i j}$. The $\delta_{z z}$ component denotes the induced magnetic field perpendicular to the molecular ring when the magnetic field is applied in the same direction, which corresponds to nucleus-independent chemical shift (NICSzz) values in the grid points. ${ }^{52,53,55}$ The magnetic response calculated for $2^{2+}, 3^{2+}$ and $3^{2-}$ is shown in Fig. 2, where the magnetic field is perpendicular and parallel with respect to the molecular ring, respectively. The long-range magnetic shielded region is seen above and below the aromatic ring. ${ }^{27-29,56}$ For 2, the region above and below the ring is deshielded suggesting that it is antiaromatic.

The calculations of the current density using the GIMIC method and the numerical integration of the ring-current strength show that 2 is antiaromatic sustaining a ring current of $-40 \mathrm{nA} \mathrm{T}^{-1}$ when the magnetic field is perpendicular to the molecular plane. The doubly charged cation $2^{2+}$ with $10 \pi$ electrons is aromatic sustaining a diatropic ring current of 18.5 $\mathrm{nA} \mathrm{T}^{-1}$, which can be compared to the ring-current strength of $12 \mathrm{nA} \mathrm{T}^{-1}$ for benzene. For $3^{2+}$ and $3^{2-}$ with $10 \pi$ and $14 \pi$ electrons, the magnetically induced current strengths are $19.3 \mathrm{nA} \mathrm{T}^{-1}$ and $18.7 \mathrm{nA} \mathrm{T}^{-1}$, respectively. Thus, the individual molecular species obey the Hückel rule for aromaticity and antiaromticity. The calculated ring-current strengths are summarized in Table 1 . The ring-current strengths of $2^{2+}$ and $3^{2+}$ are practically equal, even though $2^{2+}$ has three $\mathrm{sp}^{2} \mathrm{sp}^{2}$ vertex pairs and $3^{2+}$ four single $\mathrm{sp}^{2}$ vertices.

The annelated 2 and 3 fragments constitute the octahedral gaudiene cage with eight three-fold and six four-fold faces. The energy levels of the $72 \pi$ electrons on the surface of the allcarbon $O_{\mathrm{h}}$ structure are depicted in Fig. 3. The radial $\pi$ orbitals are labeled in terms of angular momentum functions in order to account for the shell closure as expected by the Hirsch $2(n+1)^{2}$ rule for spherical aromaticity. ${ }^{20,22}$ Hirsch's $2(n+1)^{2}$ rule can be derived by assuming that the potential due to the electronic charge density of the molecular shell is practically infinitely thin and spherical implying that orbitals without

Table 1 The calculated ring-current strengths (in $\mathrm{nA} \mathrm{T}^{-1}$ ). The diatropic (positive) and paratropic (negative) contributions are given in parenthesis

\begin{tabular}{lllr}
\hline Molecule & Symmetry & $\begin{array}{l}\text { Number of } \\
\text { electrons }\end{array}$ & Current strength \\
\hline $\mathbf{2}$ & $D_{3 \mathrm{~h}}$ & $12 \pi$ & $-34.0(4.5 /-38.5)$ \\
$\mathbf{2}^{2+}$ & $D_{3 \mathrm{~h}}$ & $10 \pi$ & $18.4(23.6 /-5.2)$ \\
$\mathbf{3}^{2+}$ & $D_{4 \mathrm{~h}}$ & $10 \pi$ & $18.7(23.4 /-4.7)$ \\
$\mathbf{3}^{2-}$ & $D_{4 \mathrm{~h}}$ & $14 \pi$ & $22.3(26.9 /-4.6)$
\end{tabular}




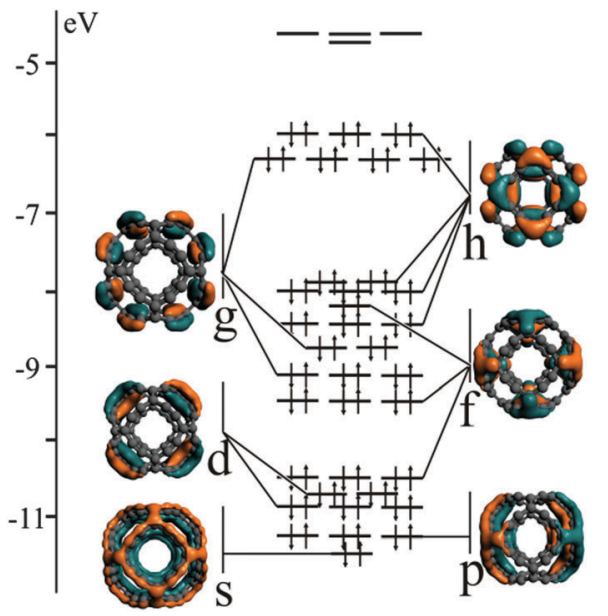

Fig. 3 The electronic structure of $C_{72}$ showing the energy levels of the 72 radial $\pi$ orbitals. The tangential $\pi$ orbitals have been neglected for clarity.

radial nodes are much lower in energy than those with a radial node. To obtain a uniform angular shape for the potential, the aufbau principle is obtained by expanding the orbitals in spherical harmonics multiplied by a nodeless radial function.

The tangential $\pi$ orbitals of the triple bonds are omitted, because they cannot contribute to the global electron delocalization. For symmetry reasons, energy levels with $l>1$ split in the $O_{\mathrm{h}}$ point group. The radial $\pi$-electron levels up to $l=5$ are shown in Fig. 3, where the HOMO is represented by the h-shell like levels, as also obtained at the B3LYP level of theory. ${ }^{17}$ The direction dependence of the magnetic shielding function can be studied by applying the external magnetic field along the symmetry axes. The isotropic representation is simpler to interpret, but it also hides some information. ${ }^{26}$ The isotropic magnetic shielding function is shown in Fig. 4.

The isosurface of the nucleus independent chemical shift function with a threshold of $-5 \mathrm{ppm}$ is located outside gaudiene at a radial distance of $1.3 \AA$ from the molecular frame. The isotropic magnetic shielding function in Fig. 4 resembles the magnetic shielding function calculated for spherical aromatic $\mathrm{C}_{60}{ }^{10+}$, which has a large and almost constant magnetic shielding inside the molecular cage. ${ }^{26}$ For gaudiene, the magnetic chemical shift in the interior of the cage is about $-30 \mathrm{ppm}$, as compared to $-80 \mathrm{ppm}$ for $\mathrm{C}_{60}{ }^{10+} \cdot{ }^{57}$ The magnetic chemical shift along the radius from the center of the molecule is about $-30 \mathrm{ppm}$ in the radial range from zero to $3.2 \AA$ and decreases to about $-10 \mathrm{ppm}$ at the molecular frame, where a larger anisotropy of the shielding function also appears, as seen in Fig. 5. At a radial distance of $7.0 \AA$ from the center, $\delta_{\text {iso }}$ is $-1.0 \mathrm{ppm}$ and it vanishes at a very long distance due to the large radius of the global ring currents around the whole gaudiene molecule. The shielding calculations show that gaudiene sustains a net diatropic current density, whereas the $\delta_{z z}$ component shown in Fig. 5 reveals some anisotropy in the magnetic shielding function, with a deshielding region of about $1 \mathrm{ppm}$ above the $\mathrm{C}=\mathrm{C}$ double bonds. The direction-dependent magnetic response is due to the slightly different current strength passing the

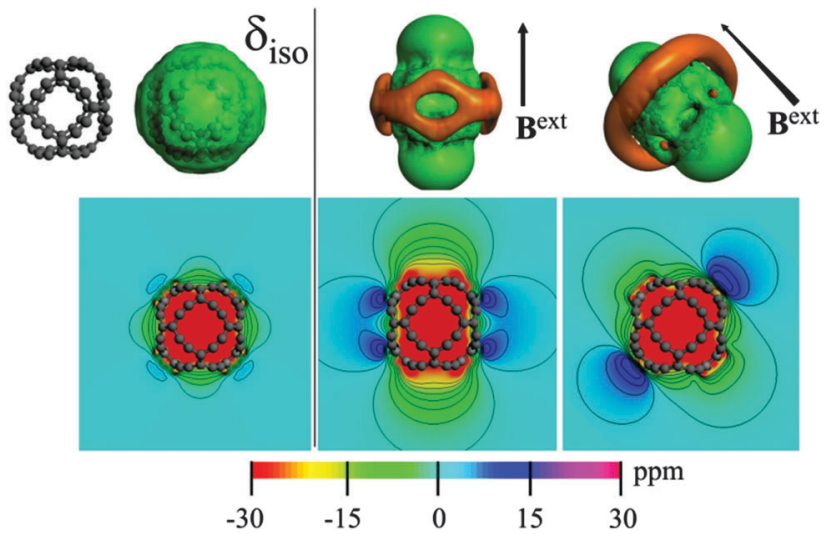

Fig. 4 Isosurface and cut-plane representations of the magnetic response of gaudiene. The isotropic and two direction specific components of the magnetic shielding functions are shown. The used isosurface values are \pm 5 ppm and the same color code is used as in Fig. 2 .

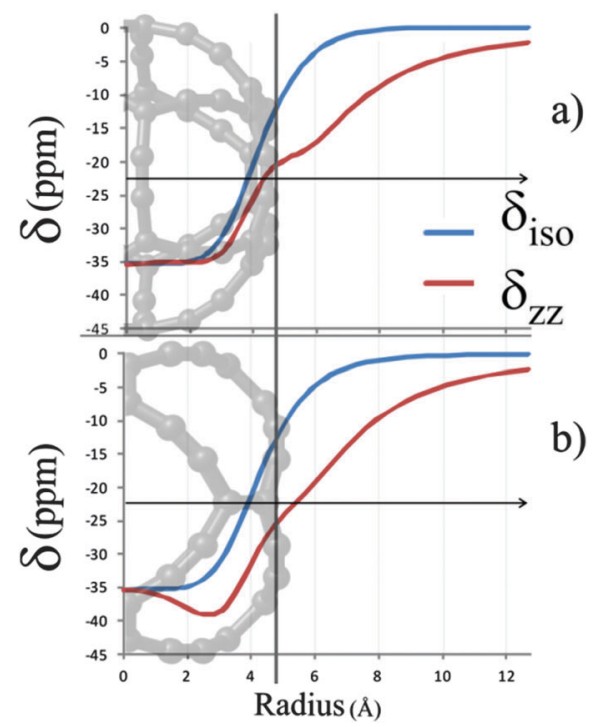

Fig. 5 The profile of the magnetic shielding function passing through the center of (a) the three-fold and (b) four-fold rings. The $z$-axis is oriented along the three-fold and four-fold symmetry axis, respectively. The origin corresponds to the center of the gaudiene cage.

chemical bonds when the annelated 2 and 3 rings of gaudiene are perpendicular to the external magnetic field.

From the cut-plane representations in Fig. 4, one can see that in the interior of the molecule the shielding function is almost direction independent, whereas at the outer part of the molecule the shielding function depends on the orientation of the external magnetic field. Thus, the magnetic shielding function of gaudiene is similar to spherical aromatic compounds by having almost isotropic shielding in the interior of the molecular cage. However, the shielding function for gaudiene is direction dependent in the outer region, which is not expected for ideal spherical aromatic species. The $\delta_{z z}$ component has a long-range shielding cone as well as a deshielding belt outside the molecular frame in the middle of the molecule. 

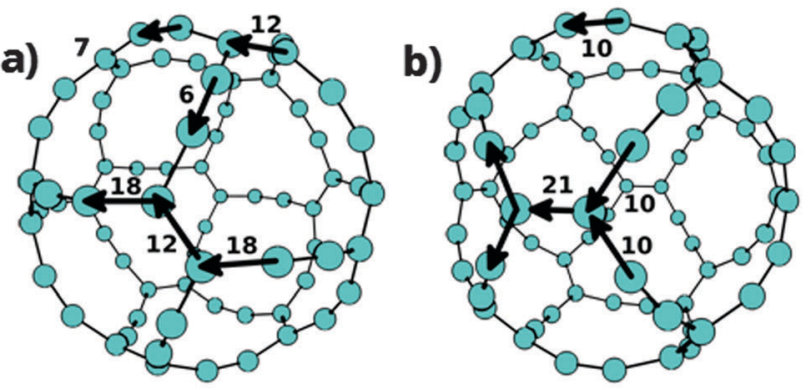

Fig. 6 The current density pathways and current strengths (in $n A T^{-1}$ ) in gaudiene as obtained for the two directions of the applied magnetic field. In (a) the magnetic field is applied along the three-fold symmetry axis and in (b) along the four-fold axis.

The calculated shielding function indicates that gaudiene sustains ring currents around the cage structure. Explicit calculations of the magnetically induced current density using the GIMIC method show that gaudiene sustains a net ring current of $40.5 \mathrm{nA} \mathrm{T}^{-1}$ around the molecular cage when the external magnetic field is oriented along the four-fold axis. A current strength of $9.7 \mathrm{nA} \mathrm{T}^{-1}$ passes along the chemical bonds of the four-membered rings that are perpendicular to the applied field. The current pathways are shown in Fig. 6. The current strength in the four-membered rings consists of diatropic and paratropic contributions of $15.4 \mathrm{nA} \mathrm{T}^{-1}$ and $-5.6 \mathrm{nA} \mathrm{T}^{-1}$, respectively. The current strength through the formal double bond at the center of the molecule is $21.0 \mathrm{nA} \mathrm{T}^{-1}$, which almost completely diatropic with $21.7 \mathrm{nA} \mathrm{T}^{-1}$, while the paratropic component is only $-0.7 \mathrm{nA} \mathrm{T}{ }^{-1}$. The current passing the formal double bond splits into two branches, whose current strengths are $10.4 \mathrm{nA} \mathrm{T}^{-1}$, and whose diatropic and paratropic contributions are $12.7 \mathrm{nA} \mathrm{T}^{-1}$ and $-2.3 \mathrm{nA} \mathrm{T}^{-1}$, respectively. Since the paratropic contribution of the double bond is much smaller, the main contribution to the paratropic current of $-2.3 \mathrm{nA} \mathrm{T}^{-1}$ is due to a local bond current. The current density forms a global ring current around the whole molecule, whereas gaudiene does not sustain any local ring currents as seen in Fig. 7.

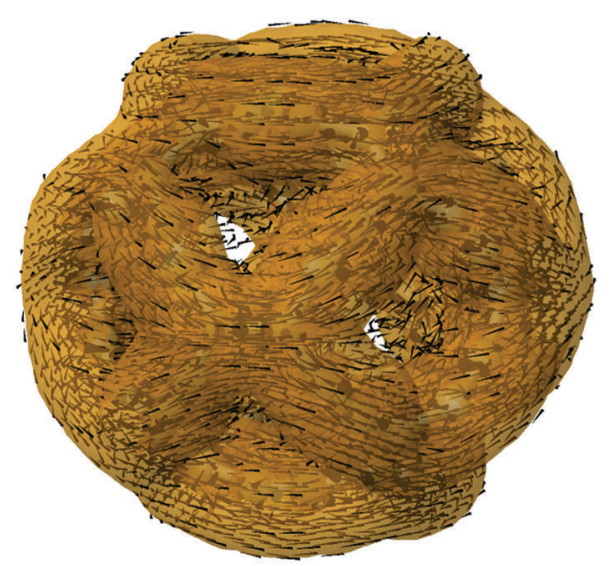

Fig. 7 The arrow plot of the magnetically induced current density shows how the ring current flows mainly along the chemical bonds.
The global ring current is $36.3 \mathrm{nA} \mathrm{T}^{-1}$, when the external field is oriented along the three-fold axis, which is somewhat weaker than the current strength of $40.5 \mathrm{nA} \mathrm{T}^{-1}$ that is obtained with the magnetic field oriented along the four-fold axis. The main contribution to the global ring current of $18.3 \mathrm{nA} \mathrm{T}^{-1}$ flows along the 'equator' in the middle of gaudiene as shown in Fig. 6. The current is dominated by the diatropic contribution of $20.4 \mathrm{nA} \mathrm{T}^{-1}$ and the paratropic current is only $-1.8 \mathrm{nA} \mathrm{T}^{-1}$. At the formal double bond of the annelated 2 rings, the ring current bifurcates into two branches whose strengths are $5.8 \mathrm{nA} \mathrm{T}^{-1}(9.0 /-3.2)$ and $12.2 \mathrm{nA} \mathrm{T}^{-1}(12.5 /-0.4)$, where the diatropic and paratropic contributions are given within parenthesis. The small paratropic contributions to the current strength along the pathway involving 24 carbons at the center of the molecule suggest that the current flow along the 'equator' is uniform. After the formal double bond, the current of $5.8 \mathrm{nA} \mathrm{T}^{-1}$ joins the $12.2 \mathrm{nA} \mathrm{T}^{-1}$ current continuing along the equator. The current strength in the double bond of the annelated 2 rings at the poles is $12.2(15.5 /-3.3)$ and along the triple bonds it is $6.8 \mathrm{nA} \mathrm{T}^{-1}(12.9 /-6.1)$. The current strengths differ by $10 \%$ for the two orientations of the magnetic field, which is the reason for the slightly direction-dependent magnetic shielding function. The molecular rings are too large for a uniform current transport across them. Instead, the ring current flows mainly along the chemical bonds as shown in Fig. 6 and 7 .

\section{Conclusions}

The studied cavernous gaudiene carbocage $\left(\mathrm{C}_{72}\right)$ of $O_{\mathrm{h}}$ symmetry has two main symmetry axes with three- and four-fold faces. The magnetic response of gaudiene has been investigated by exposing it to external magnetic fields. Applying the magnetic field along the two symmetry axes leads to different pathways of the induced ring current and to somewhat different current strengths passing along the chemical bonds. The external magnetic field results in a net diatropic current density flow in the almost spherical structure of the molecular frame. The induced current density flows mainly along the chemical bonds that are perpendicularly oriented with respect to the applied field constituting the global ring current around the carbocage. The strength of the global ring current differs by only $10 \%$ when the magnetic field is applied along the two symmetry axes, suggesting that the current flow is almost as isotropic as for ideal spherical aromatic structures. The induced magnetic field exhibits a long-range shielding cone parallel to the direction of the external magnetic field, whereas a small deshielding region is located perpendicularly to the field outside the molecular frame. The gaudiene cage uniformly shields the magnetic field inside the cage leading to an isotropic magnetic shielding function in the interior of the molecule. Thus, the anisotropic magnetic response appears only at the exterior of the molecular cage. Even though the number of $\pi$ electrons on the surface of the molecular cage and the current strength fulfill the criterion for spherical aromaticity, the anisotropicity of current density and the mixing of the energy levels suggest that gaudiene cannot 
be considered to be an ideal spherical aromatic molecule. $\mathrm{C}_{72}$ is an aromatic molecule with strong almost isotropic global ring currents along the chemical bonds leading to isotropic diamagnetic shielding inside the molecular frame and a region with weak paramagnetic shielding outside the molecule.

\section{Acknowledgements}

The authors are thankful for the financial support given by FONDECYT 1140359, Project Millennium RC120001, and the Academy of Finland through projects 266227 and 275845. DS thanks the Swedish Cultural Foundation in Finland and Magnus Ehrnrooth Foundation for financial support. Computational resources have been provided by CSC - the Finnish IT Center for Science and by FONDECYT funds.

\section{References}

1 H. W. Kroto, J. R. Heath, S. C. O'Brien, R. F. Curl and R. E. Smalley, Nature, 1985, 318, 162-163.

2 R. Taylor, J. P. Hare, A. K. Abdul-Sada and H. W. Kroto, J. Chem. Soc., Chem. Commun., 1990, 1423-1424.

3 H. W. Kroto, Angew. Chem., Int. Ed. Engl., 1992, 31, 111-129.

4 K. M. Kadish and R. S. Ruoff, Fullerenes: Chemistry, Physics, and Technology, Wiley-Interscience, New York, 2000.

5 A. Hirsch and M. Brettreich, Fullerenes: Chemistry and Reactions, Wiley-VCH, Weinheim, 2005.

6 E. Sheka, Fullerenes: Nanochemistry, Nanomagnetism, Nanomedicine, Nanophotonics, CRC Press, 2011.

7 A. Rodríguez-Fortea, S. Irle and J. M. Poblet, Wiley Interdiscip. Rev.: Comput. Mol. Sci., 2011, 1, 350-367.

8 M. Zheng, Y. Liu, S. Zhao, W. He, Y. Xiao and D. Yuan, Inorg. Chem., 2010, 49, 8674-8683.

9 R. O. Loutfy and S. Katagiri, in Perspectives of Fullerene Nanotechnology, ed. E. Ōsawa, Springer, Netherlands, Dordrecht, 2002, pp. 357-367.

10 K. T. Lee, Y. S. Jung and S. M. Oh, J. Am. Chem. Soc., 2003, 125, 5652-5653.

11 Y. Chabre, D. Djurado, M. Armand, W. R. Romanow, N. Coustel, J. P. McCauley, J. E. Fischer and A. B. Smith, J. Am. Chem. Soc., 1992, 114, 764-766.

12 Y. Song, Y. Li and X. Xia, Electrochem. commun., 2007, 9, 201-205.

13 Y. H. Ng, S. Ikeda, T. Harada, S. Higashida, T. Sakata, H. Mori and M. Matsumura, Adv. Mater., 2007, 19, 597-601.

14 O. V Pupysheva, A. A. Farajian and B. I. Yakobson, Nano Lett., 2008, 8, 767-774.

15 H. Hoppe and N. S. Sariciftci, J. Mater. Chem., 2006, 16, 45-61.

16 A. Montellano, T. Da Ros, A. Bianco and M. Prato, Nanoscale, 2011, 3, 4035-4041.

17 D. Sundholm, Phys. Chem. Chem. Phys., 2013, 15, 9025-9028.

18 D. Sundholm, L. N. Wirz and P. Schwerdtfeger, Nanoscale, 2015, 7, 15886-15894.

19 Z. Chen and R. B. King, Chem. Rev., 2005, 105, 3613-3642.
20 A. Hirsch, Z. Chen and H. Jiao, Angew. Chem., 2000, 39, 3915-3917.

21 Z. Chen, H. Jiao, D. Moran, A. Hirsch, W. Thiel and P. von Ragué Schleyer, J. Phys. Org. Chem., 2003, 16, 726-730.

22 M. Bühl and A. Hirsch, Chem. Rev., 2001, 101, 1153-1184.

23 H. Fliegl, S. Taubert, O. Lehtonen and D. Sundholm, Phys. Chem. Chem. Phys., 2011, 13, 20500-20518.

24 J. Jusélius, D. Sundholm and J. Gauss, J. Chem. Phys., 2004, 121, 3952-3963.

25 D. Sundholm, H. Fliegl and R. J. F. Berger, Wiley Interdiscip. Rev.: Comput. Mol. Sci., 2016, DOI: 10.1002/wcms.1270.

26 A. Muñoz-Castro, Chem. Commun., 2015, 51, 10287-10290.

27 R. Islas, T. Heine and G. Merino, Acc. Chem. Res., 2012, 45, 215-228.

28 G. Merino, T. Heine and G. Seifert, Chemistry, 2004, 10, 4367-4371.

29 M. Kaupp, M. Bühl and V. G. Malkin, Calculation of NMR and EPR Parameters: Theory and Applications, John Wiley \& Sons, 2006.

30 S. Klod and E. Kleinpeter, J. Chem. Soc., Perkin Trans. 2, 2001, 1893-1898.

31 E. Kleinpeter, S. Klod and A. Koch, J. Org. Chem., 2008, 73, 1498-1507.

32 E. Kleinpeter, S. Krüger and A. Koch, J. Phys. Chem. A, 2015, 119, 4268-4276.

33 M. Baranac-Stojanović and E. Kleinpeter, J. Org. Chem., 2011, 76, 3861-3871.

34 A. Muñoz-Castro, J. Phys. Chem. C, 2012, 116, 17197-17203.

35 A. Muñoz-Castro, Chem. Phys. Lett., 2013, 555, 282-285.

36 H. scm, co. ADF, Vrije Universiteit, Amsterdam, The Netherlands, SCM.

37 G. Te Velde, F. M. Bickelhaupt, E. J. Baerends, C. Fonseca Guerra, S. J. A. van Gisbergen, J. G. Snijders and T. Ziegler, J. Comput. Chem., 2001, 22, 931-967.

38 A. D. Becke, Phys. Rev. A: At., Mol., Opt. Phys., 1988, 38, 3098-3100.

39 J. P. Perdew, Phys. Rev. B: Condens. Matter Mater. Phys., 1986, 33, 8822-8824.

40 S. H. Vosko, L. Wilk and M. Nusair, Can. J. Phys., 1980, 58, 1200-1211.

41 K. Wolinski, J. F. Hinton and P. Pulay, J. Am. Chem. Soc., 1990, 112, 8251-8260.

42 G. Schreckenbach and T. Ziegler, J. Phys. Chem., 1995, 99, 606-611.

43 S. K. Wolff, T. Ziegler, E. van Lenthe and E. J. Baerends, J. Chem. Phys., 1999, 110, 7689-7698.

44 F. Furche, R. Ahlrichs, C. Hättig, W. Klopper, M. Sierka and F. Weigend, Wiley Interdiscip. Rev.: Comput. Mol. Sci., 2014, 4, 91-100.

45 TURBOMOLE V7.0 2015, a development of University of Karlsruhe and Forschungszentrum Karlsruhe $\mathrm{GmbH}$, 1989-2007, TURBOMOLE GmbH, since 2007; available from http://www.turbomole.com.

46 A. Schäfer, C. Huber and R. Ahlrichs, J. Chem. Phys., 1994, 100, 5829-5835.

47 F. Weigend and R. Ahlrichs, Phys. Chem. Chem. Phys., 2005, 7, 3297-3305. 
48 K. Eichkorn, O. Treutler, H. Öhm, M. Häser and R. Ahlrichs, Chem. Phys. Lett., 1995, 240, 283-290.

49 J. Jusélius and D. Sundholm, Phys. Chem. Chem. Phys., 2001, 3, 2433-2437.

50 J. Jusélius and D. Sundholm, Phys. Chem. Chem. Phys., 2008, 10, 6630-6634.

51 T. Heine, C. Corminboeuf and G. Seifert, Chem. Rev., 2005, 105, 3889-3910.

52 P. R. von Schleyer and H. Jiao, Pure Appl. Chem., 1996, 68, 209-218.
53 H. Fallah-Bagher-Shaidaei, C. S. Wannere, C. Corminboeuf, R. Puchta and P. V. R. Schleyer, Org. Lett., 2006, 8, 863-866.

54 J. A. Pople and K. G. Untch, J. Am. Chem. Soc., 1966, 88, 4811-4815.

55 P. von, R. Schleyer, C. Maerker, A. Dransfeld, H. Jiao and N. J. R. van Eikema Hommes, J. Am. Chem. Soc., 1996, 118, 6317-6318.

56 J. A. Pople, J. Chem. Phys., 1956, 24, 1111.

57 M. P. Johansson, D. Sundholm and J. Vaara, Angew. Chem., Int. Ed., 2004, 43, 2678-2681. 